\title{
Expression and Significance of MMPs in Synovial Fluid, Serum and PBMC Culture Supernatant Stimulated by LPS in Osteoarthritis Patients With or Without Diabetes
}

\author{
Authors \\ Simin Luo ${ }^{1,2^{*}}$, Qiping Shi ${ }^{*}$, Junyuan Chen ${ }^{1,2^{*}}$, Huajun Wang ${ }^{1,2}$, Wenrui Wu ${ }^{1,2}$, Zhengang Zha1, 2
}

Affiliations

1 The First Affiliated Hospital, Jinan University, Guangzhou, China

2 Institute of Orthopaedic Disease Research, Jinan University, Guangzhou, China

Key words

Osteoarthritis, Type 2 Diabetes mellitus, Matrix metalloproteinase, Peripheral Blood Mononuclear Cell

$\begin{array}{lr}\text { received } & 08.06 .2017 \\ \text { revised } & 08.10 .2017 \\ \text { accepted } & 30.10 .2017\end{array}$

\section{Bibliography}

DOI https://doi.org/10.1055/s-0043-122223

Published online: 21.12.2017

Exp Clin Endocrinol Diabetes 2019; 127: 195-202

(C) J. A. Barth Verlag in Georg Thieme Verlag KG Stuttgart . New York

ISSN 0947-7349

\section{Correspondence}

Prof. Zhengang Zha

The First Affiliated Hospital, Jinan University

613 Huangpu Avenue West, Guangzhou

China

Tel.: + 86/20/38688 617, Fax: + 86/20/38688 000

zhzgg@vip.163.com

\section{ABSTRACT}

Objective Patients with Type 2 Diabetes mellitus (T2DM) are prone to osteoarthritis (OA). Matrix metalloproteinases (MMPs), an essential modulator in cartilage matrix homeostasis, increase in T2DM and OA. We aimed to ascertain the expression difference of MMPs and function in mononuclear cells after stimulating by lipopolysaccharide (LPS) in OA patients with or without diabetes.

Methods 30 knee OA patients without T2DM (OA group), 20 knee OA patients with T2DM (DM-OA group) and 5 healthy volunteers recruited as control were enrolled from January 2016 to January 2017. The expression levels of MMPs in both serum and synovial fluid were initially detected in three groups by enzyme-linked immunosorbent assay (ELISA). After stimulation of peripheral blood mononuclear cell (PBMC) with LPS, the release of MMPs were determined and evaluated.

Results The expression of MMP- $1,-7,-8,-9,-10$ and -12 in synovial fluid in DM-OA group were significantly higher than in OA group and healthy control. The expression of MMP-1 and -7 in serum were highest in DM-OA group. LPS significantly promotes the production of MMP-1, $-8,-9$ and -10 in PBMC of each group after $4 \mathrm{~h}$ stimulation. It is worth to note that the LPSstimulated MMP-8 and -9 elevations were more prominent in DM-OA group compared with their counterparts.

Conclusion High levels of MMP- $1,-7,-8,-9,-10$, and -12 in the synovial fluid might be one of important reasons that diabetes patients are more frequently suffered from OA. Inflammation-induced malfunction of mononuclear cells would stimulate MMP-8 and -9 secretion to various extents.

\section{Introduction}

Osteoarthritis (OA) is the most common form of chronic joint disorders, an ongoing biological degenerative joint disease with erosion of articular cartilage, breakdown of subchondral bone, and symptoms of inflammation in synovium, causing significant functional impairment and disability in elderly [1]. OA may occur in different kinds of joints, while knee involvement is the most common one. Female, increasing age, obesity, prior joint damage, genetic predisposition, abnormal mechanical stress of joints, and endocrine fac-

* Contribute equally to this paper. tors are deemed to participate in the occurrence and progression of $\mathrm{OA}[2,3]$. Recently, growing evidence suggests that metabolic factors play a leading role in both pathogenesis and progression of $\mathrm{OA}$ $[3,4]$. Proteolytic enzymes contribute to degrading of collagens and proteoglycans. Both are components of the extracellular matrix $(E C M)$, give rise to brittle cartilage that is prone to mechanical damage. As a family of zinc-dependent proteolytic enzymes, matrix metalloproteinases (MMPs) takes part in the degeneration of targeted proteins through the cleavage of internal peptide bonds [5].

Normally, chondrocytes express multifarious proteolytic enzymes such as aggrecanases and MMPs to regulate matric metabolism, which is responsible for cartilage remodeling [6]. On the con- 
trary, chondrocytes and synovial fibroblasts secrete more enzymes, resulting in aberrant cartilage destruction in OA. Substantial researches have verified the relationship between MMPs and OA $[7,8]$. MMP-1, $-2,-8,-9$ and -13 protein expression levels have been declared to be correlated to the severity of the disease $[9,10]$. This phenomenon has attracted increased attention that MMPs as the promoter of cartilage degradation, activating each other, producing "waterfall-like" amplification action and conducting cartilage damage $[11,12]$.

Associating with OA, type 2 diabetes mellitus (T2DM) could be an independent and prominent risk factor $[13,14]$, leading to concepts of diabetic OA phenotype or diabetes-accelerated OA [15]. However, the regulating mechanism of diabetic in OA remains elusive. Maintaining tissue allostasis, MMPs are altered or increased in both T2DM and OA. However, few studies evaluate the presence of MMPs in OA patients with diabetes. One purpose of this study is to clarify the role of MMPs in OA patients, with or without diabetes, to investigate correlations between MMPs and pathogenesis of diabetic $O A$.

Lipopolysaccharide (LPS) is one of the structural components of the outer membrane of Gram-negative bacteria. LPS can induce inflammatory response in human peripheral blood mononuclear cells (PBMCs), eventually leads to expression and production of many pro-inflammatory cytokines, such as interleukin (IL) - $1 \beta$, IL-6, tumor necrosis factor- $\alpha$ (TNF $\alpha$ ) and MMPs. Recent studies have shown that a low-grade systemic inflammatory process such as diabetes may increase the risk of OA by two-fold [16]. Another purpose of this study is to research whether OA patients with diabetes had a much higher inflammatory response than common OA patients.

\section{Materials and Methods}

\section{Participants and study design}

From January 2016 to January 2017, 30 knee OA patients without T2DM (OA group) and 20 knee OA patients with T2DM (DM-OA group) admitting at our hospital were enrolled. Besides, 5 healthy volunteers without OA and T2DM were recruited as a healthy control (HC) group. OA was diagnosed by clinical and radiological evaluation based on the American College of Rheumatology criteria. T2DM was diagnosed according to the classic American Diabetes Association criteria(fasting plasma glucose $\geq 7.0 \mathrm{mmol} / \mathrm{L}$ and glycated hemoglobin $(\mathrm{HbA} 1 \mathrm{c}) \geq 6.5 \%$ ) [17], or a clinical diagnosis of T2DM with ongoing antidiabetic treatment.

Patients with evident joint injury, previous knee surgery or with inflammatory arthritis were excluded from the study. Also, those who received intra-articular corticosteroid or hyaluronic acid injections within 3 months or with serious cardiovascular disease (CVD), cerebrovascular conditions within 6 months before study enrollment were excluded too. Diabetic patients were excluded if they had a history of ketoacidosis or suffering from unstable or rapidly progressive diabetic retinopathy, nephropathy, or neuropathy.

This study conforms to the provisions of the Declaration of Helsinki and was approved by the hospital medical ethics committees, and informed consent was obtained from all participants before the study.

\section{Sample collection}

Synovial fluid harvesting was performed prior to total knee arthroplasty (TKA) or utilizing joint cavity paracentesis, strictly avoiding hemarthrosis. Before collecting synovial fluid samples of controls, $5 \mathrm{~mL}$ saline was injected into the articular cavity and rinsed repeatedly. Then the flushing fluid was sequentially aspirated, sub-packaged and stored in liquid nitrogen for later analysis. Fasting blood samples were collected from a peripheral vein and drawn into vacuum blood tubes containing lithium heparin. The samples were centrifuged or $20 \mathrm{~min}$ at $1,000 \times \mathrm{g}$ at $4^{\circ} \mathrm{C}$. The supernatant was collected and stored at $-80^{\circ} \mathrm{C}$ until use.

\section{ELISA assay}

Serum and synovial fluid expression levels of MMP-1, $-2,-3,-7,-8$, $-9,-10,-12,-13$ in three groups were detected by ELISA kit (Elabscience, Wuhan, China) according to the manufacturer's protocol, respectively. Briefly, the micro ELISA plate has been pro-coated with an antibody specific to MMPs, and the appropriate diluent serum or synovial fluid samples were added in each microplate well, followed with incubation for $90 \mathrm{~min}$ at $37^{\circ} \mathrm{C}$. After washing with wash buffer, the corresponding secondary antibody was applied. After incubation for $60 \mathrm{~min}$, the plates were washed again. The optical density was measured spectrophotometrically at a wavelength of $450 \mathrm{~nm}$. The concentration of MMPs was calculated from a standard curve.

\section{PBMC collection and stimulation}

PBMCs were isolated by Ficoll gradient centrifugation from all participants, diluted with equal amount of PBS, overlaid on Ficoll medium slowly, and centrifuged at $600 \mathrm{~g}$ for $30 \mathrm{~min}$ at $20^{\circ} \mathrm{C}$. The PBMCs band was aspirated, washed twice with PBS, and re-suspended in cell freezing medium (90\% FBS, $10 \%$ DMSO) before gradient cooling being stored in liquid nitrogen for later use.

After all the PBMCs samples had been collected, the PBMCs were resuspended in medium firstly, calculated the number to $2 \times 10^{5} /$ $\mathrm{ml}$, then stimulated with LPS (a TLR4 ligand) at $100 \mathrm{ng} / \mathrm{ml}$ for $4 \mathrm{~h}$, and the cell culture supernatants were gathered before slit charging and being stored at $-80^{\circ} \mathrm{C}$. And the MMPs in the supernatants were analyzed by Elisa Kit (Elabscience Biotechnology Co.,Ltd)

\section{Statistical analysis}

All statistical analyses were performed using IBM SPSS Statistic 19.0 (New York, NY). Continuous variables were expressed as the mean \pm standard deviation (SD). Statistically significant differences between multiple groups were determined analyzing variance (ANOVA) in conjunction with Tukey's multiple comparison. A statistical significance was defined when $\mathrm{P}<0.05$.

\section{Results}

\section{Descriptive statistics}

The sample characteristics, including the general data and the laboratory results of hospital are provided in > Table 1 . Our results showed that LDL concentration, fasting glucose and $\mathrm{HbA} 1 \mathrm{c}$ in $D M-O A$ group were higher than in OA group $(P<0.05)$. The age, $c-$ reactive peptide and erythrocyte sedimentation rate in $\mathrm{DM}-\mathrm{OA}$ and OA group were higher than in control $(P<0.05)$. 
- Table 1 Clinical and laboratory characteristics of study subjects.

\begin{tabular}{|c|c|c|c|}
\hline & control & OA & diabetic OA \\
\hline$n$ & 5 & 20 & 30 \\
\hline $\operatorname{Sex}(M / F)$ & $2 / 3$ & $3 / 17$ & $7 / 23$ \\
\hline Age (years) & $37.6 \pm 9.8$ & $67.3 \pm 8.7^{*}$ & $70.4 \pm 8.3^{*}$ \\
\hline Body mass index & $23.15 \pm 3.98$ & $25.26 \pm 3.09$ & $26.83 \pm 5.62$ \\
\hline OA Duration (years) & - & $7.14 \pm 3.88$ & $8.43 \pm 4.70$ \\
\hline $\begin{array}{l}\text { Arthritic joints } \\
\text { (unilateral/bilateral) }\end{array}$ & - & $8 / 12$ & $11 / 19$ \\
\hline Fasting glucose (mmol/L) & $5.13 \pm 0.65$ & $5.69 \pm 0.92$ & $6.36 \pm 1.02^{\#}$ \\
\hline HbA1c (\%) & $4.58 \pm 0.79$ & $5.12 \pm 0.91$ & $6.68 \pm 1.05^{\# *}$ \\
\hline LDL cholesterol(mg/dL) & $2.45 \pm 0.87$ & $2.73 \pm 0.75$ & $3.37 \pm 1.18^{\#}$ \\
\hline HDL cholesterol(mg/dL) & $1.07 \pm 0.08$ & $1.29 \pm 0.29$ & $1.41 \pm 0.38$ \\
\hline Triglycerides(mg/dL) & $0.97 \pm 0.34$ & $1.36 \pm 0.50$ & $1.52 \pm 0.76$ \\
\hline Total cholesterol(mg/dL) & $4.05 \pm 2.56$ & $4.49 \pm 1.23$ & $5.44 \pm 1.36$ \\
\hline C-reactiv peptide(mg/L) & $2.26 \pm 1.78$ & $4.99 \pm 3.68^{*}$ & $5.67 \pm 4.97^{*}$ \\
\hline Erythrocyte sedimentation rate(mm/h) & $13.1 \pm 6.48$ & $28.45 \pm 17.26^{*}$ & $32.65 \pm 18.11^{*}$ \\
\hline
\end{tabular}

\section{Basal expression of MMPs in healthy control, OA and DM-OA human synovial fluid}

The levels of MMPs- $1,-2,-3,-7,-8,-9,-10,-12,-13$ in synovial fluid were measured by ELISA. The expression of MMP-1 was significantly higher in OA and DM-OA group than that in healthy control (both $\mathrm{P}<0.0001)$. Moreover, MMP-1 in DM-OA group was approximately 2.5 times higher than that in OA group $(\mathrm{P}<0.0001$, > Fig. 1a).

MMP-2, -3 and -13 protein levels were significantly higher in $\mathrm{OA}$ and $\mathrm{DM}-\mathrm{OA}$ group than healthy control (all $\mathrm{P}<0.0001)$. However, there was no significant difference between $\mathrm{OA}$ and $\mathrm{DM}-\mathrm{OA}$ group in MMP- $2,-3$ and $-13(P=0.177,0.999,0.974$, respectively. - Fig. 1b, c and i).

The levels of MMP-7, -10 and -12 protein in OA and DM-OA group were substantially higher than those in healthy control (all $P<0.0001$, except MMP-12 in OA group, $P=0.011$ ). Surprisingly, the difference in MMP-7, -10 and -12 protein levels between OA and DM-OA group was statistically significant (all $\mathrm{P}<0.0001$, - Fig. $1 d, g$ and $h$ ).

On average, MMP-8 and -9 protein levels were approximately 25 and 9 times higher in DM-OA group than in OA group, respectively. Nevertheless, the difference between OA group and healthy control did not reach statistical significance $(P=0.958$ and 0.984 , $\checkmark$ Fig. 1e and f).

\section{Basal expression of MMPs in healthy control, OA and DM-OA human serum}

The levels of MMP-1, $-3,-7,-12$ and -13 protein in serum in OA and DM-OA group were obviously higher than in control $(P<0.05)$. Moreover, the levels of MMP- 1 and -7 were the highest in DM-OA group $(P=0.001$ and $P<0.0001$, $>$ Fig. $2 a$ and d $)$. Nonetheless, MMP-3 in OA group was significantly higher than those in DM-OA group $(P<0.0001$, $>$ Fig. $2 c)$, and there were no significance difference between OA and DM-OA group in MMP- 12 and $-13(P=0.930$ and 0.702 , $>$ Fig. $2 \mathrm{~h}$ and i).
However, the levels of MMP-2 protein was the highest in OA group $(P<0.0001, \vee$ Fig. 2 b $)$, and there were no differences between MMP-8, -9 and -10 in these three groups $(P=0.160,0.996$ and 0.990 , respectively. $\triangleright$ Fig. $\mathbf{2 e}$, $\mathbf{f}$ and $\mathbf{g}$ ).

\section{Role of PBMC in modulating MMP expression profiles induced by LPS in healthy control, OA and DM-OA human cell culture supernatant}

PMBC failed to secrete MMP-2, $-3,-7,-12,-13$ before and after $4 \mathrm{~h}$ stimulation with LPS, while analysis of MMPs production in the cell supernatant by ELISA revealed significant augmentations of MMP$1,-8,-9,-10$ after $4 \mathrm{~h}$ of stimulation with LPS compared to those before stimulation, respectively (all $\mathrm{P}<0.0001$, $>$ Fig. 3 ).

As showed in > Fig. 3a, in PMBC culture supernatant, secretion of MMP-1 was higher in OA and DM-OA group than for healthy control before and after stimulation with LPS $(P<0.05)$.

There was no difference in the production of MMP- 8 among the three groups in the supernatant of unstimulated cells $(P=0.882$, - Fig. 3b), and the levels of MMP-9 in OA and DM-OA groups were markedly and significantly higher than those in healthy control (both $\mathrm{P}<0.0001$, > Fig. 3 c), whereas there was a significant different after $4 \mathrm{~h}$ stimulation with LPS in MMP-8 and -9 (both $\mathrm{P}<0.0001$ ). The supernatant of PBMC in DM-OA group exhibited much higher level of MMP- 8 and -9 (both $P<0.0001$, $>$ Fig. 3 b and c).

Secretion of MMP- 10 was higher in OA and DM-OA groups than healthy control in unstimulated state (both $\mathrm{P}<0.0001$ ). After $4 \mathrm{~h}$ of LPS stimulation, MMP-10 protein levels increased to $543.44 \pm 15.38 \mathrm{pg} / \mathrm{mL}(\mathrm{HC}), 559.16 \pm 42.41 \mathrm{pg} / \mathrm{mL}(\mathrm{OA})$, and $550.72 \pm 58.16 \mathrm{pg} / \mathrm{mL}$ (DM-OA) ( $\vee$ Fig. 3d), respectively. This increase in the expression of MMP-10 was not significant for the three groups $(P=0.711)$.

The present study demonstrate that there is significantly more MMP-8, -9 secreted by PBMC after LPS activation in DM-OA group. Synchronous finding was observed in synovial fluid. 


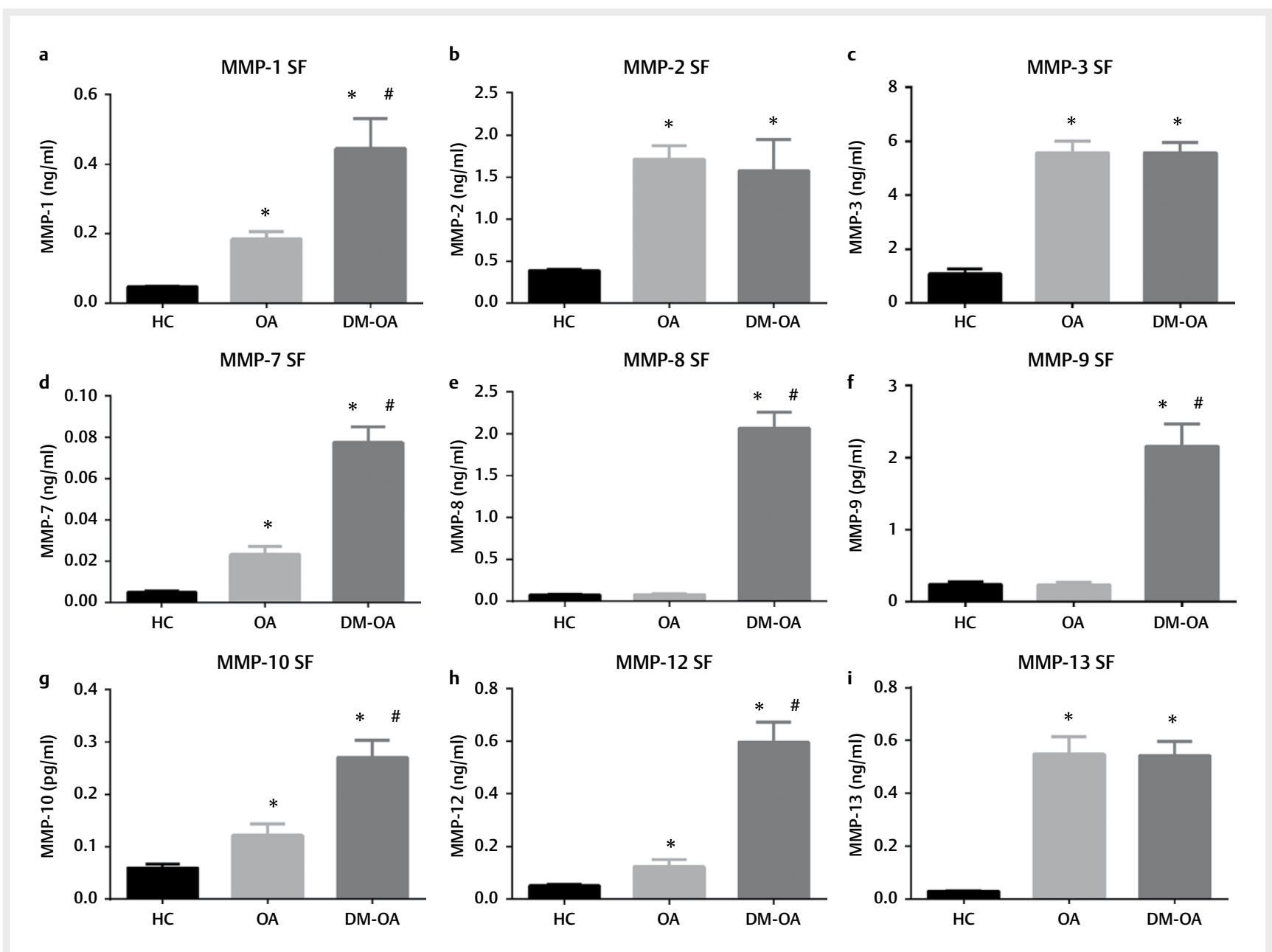

-Fig. 1 Synovial fluid expression level of MMPs in healthy controls (HC group), OA patients without T2DM (OA group) and OA patients with T2DM (DM-OA group) were measured by ELISA. aMMP-1; bMMP-2; cMMP-3; dMMP-7; e MMP-8; f MMP-9; g MMP-10; h MMP-12; i MMP-13. Each bar represents the mean $\pm S D$. ${ }^{*} \mathrm{P}<0.05$ vs. healthy controls. $\# \mathrm{P}<0.05$ vs. $\mathrm{OA}$ alone. $\mathrm{p}$ values result from a Tukey's multiple comparison test.

\section{Discussion}

Articular cartilage, a specialized connective tissue, consists of chondrocytes and ECM. Chondrocytes are unique cellular components embedded in ECM that maintain homeostasis between synthesis and catabolism under normal physiological environment [18]. Cartilage integrity crucially facilitates distributing loads and ensures a near-frictionless motion of joints.

$\mathrm{OA}$, one of age-related skeletal diseases, results in the progressive damage of the articular cartilage owing to inflammation of synovium and the consequent imbalance between catabolism and anabolism of synoviocytes and chondrocytes. MMPs take part in the process of tissue remodeling, either physiological or pathological [19]. Plenty of studies revealed that after articular injury, MMPs, especially MMP-2 and MMP-9, have been participated in the disease progression of OA [20]. Catterall et al. [21] reported that MMP-3 concentration distinctly elevates in synovial fluid within 3 weeks after ACL injury whereas Tchetverikov [22] demonstrated that both MMP-1 and MMP-3 synchronously rose 4 days after collateral ligaments of knee injury. Several MMPs and degraded aggrecan concentrations in synovial fluid were increased after an acute intraar- ticular fracture compared with a uninjured joint in the same patient, and maintained in the high level at a secondary time [23].

A few researches indicated that there was a distinct positive correlation between plasma glucose levels and radiological evidence of OA [24]. Several studies support that OA is more prevalent and severe in T2DM patients [13, 25]. OA is increasingly regarded as a "metabolic disorder disease" associated with obesity, diabetes [26].

The cardinal aim of this study was to ascertain the expression differences of MMPs in synovial fluid and plasma as well as release of MMPs by mononuclear cells after stimulation with LPS in OA patients with or without diabetes. We found that OA patients with diabetes have high levels of MMP-1, -7, -8, -9, -10, and -12 in synovial fluid. These MMPs directly impair cartilage integrity by upsetting modulating balance of anabolism and catabolism.

The results are presented in $\mathbf{- F i g s .} \mathbf{1} \mathbf{i}$ and $\mathbf{\nabla} \mathbf{2}$ are consistent with previous studies [27] that MMP-13 expression is higher in OA than in normal synovial fluid and serum. In addition, our data revealed that MMP-13 expression in the synovial fluid of OA patients with or without diabetes did not differ significantly. Rosa et al. [28] reported that the expression of MMP-13 and collagen I was not af- 


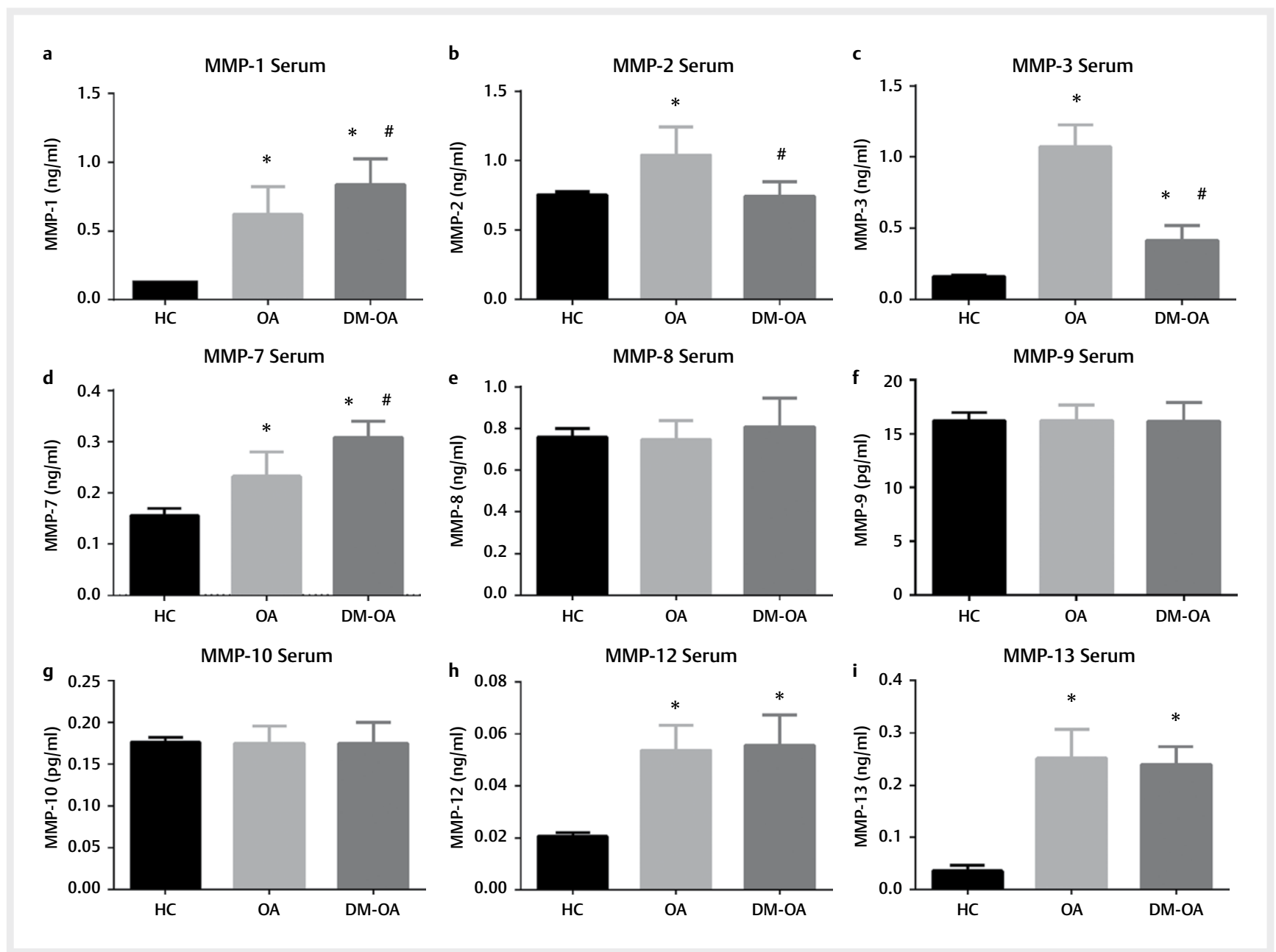

- Fig. 2 MMPs serum expression level in HC, OA and DM-OA groups were measured by ELISA. aMMP-1; bMMP-2; cMMP-3; dMMP-7; e MMP-8; $\mathbf{f}$ MMP-9; $g$ MMP-10; $\mathbf{h}$ MMP-12; i MMP-13. Each bar represents the mean \pm SD. ${ }^{*} \mathrm{P}<0.05$ vs. healthy controls. \#P<0.05 vs. OA alone. $\mathrm{p}$ values result from a Tukey's multiple comparison test.

fected by high glucose in normal chondrocytes. This suggests that MMP-13 is not a major factor of diabetes susceptibility to OA. Indeed, as a key collagenase, MMP-13 expression has been shown to be augmented in OA cartilage [29], suggesting a significant role in cartilage degradation as it preferentially cleaves type II collagen fibers [30].

Generally, MMP-2 proteins are gelatinases of the MMP family [20]. MMP-2 are secreted extracellularly, and these gelatinases cleave other targets, including growth factors, ECM, and cytokines, which results in the release of these ligands, which in turn activate major signaling pathways that are involved in cell growth, invasion, inflammation, and migration [31]. Previous study [32] showed that, MMP-3 levels in OA patient's blood and synovial fluid were higher than that in healthy people, and the increment was consistent with the extent of cartilage damage. MMP-2 expression in the synovial fluid of OA and DM-OA patients was substantially greater than that of healthy control, while the difference in serum did not differ significantly between DM-OA patients and healthy control. Moreover, MMP-2 and MMP-3 in serum were higher in OA patients than in DM-OA patients. We anticipated that upregulated MMP-2, -3 secretion might have unfavorable effects on cartilage integrity, but it is not the cause of diabetes susceptibility to OA. There may be a factor of diabetes that limits the MMP-2, -3 secretion in serum, potentially a protective mechanism exists. If MMP-2, -3 secretion is to be inhibited from chondrocytes and synovial fibroblasts, it may be possible to avoid the occurrence of OA.

MMP-7 protein was detected in synovial fluid and serum, we found that the expression of MMP-7 protein in DM-OA patients was higher than that in $\mathrm{OA}$ patients and healthy control. According to previous studies, high glucose concentrations favor catabolism in primary human chondrocytes and facilitate the development and progression of OA [28]. Elevated MMP-7 has been found to increase cartilage degradation and play a role in inflammatory joint disease [33]. Therefore, our results indicated that increasing secretion of MMP-7 in serum and synovial fluid due to diabetes mediates inflammatory OA-like effects in articular cartilage.

Further detailed studies are warranted to understand the roles of the MMP- 1 and -9 proteins in the pathogenesis of OA as a prelude to clinical application of the knowledge gained [34]. Our results indicated that MMP-1,-8, $-9,-10$ and -12 expressions were highest in DM-OA patients in synovial fluid. Interestingly, we found unexpected results that serum did not exhibit such statistically sig- 
a

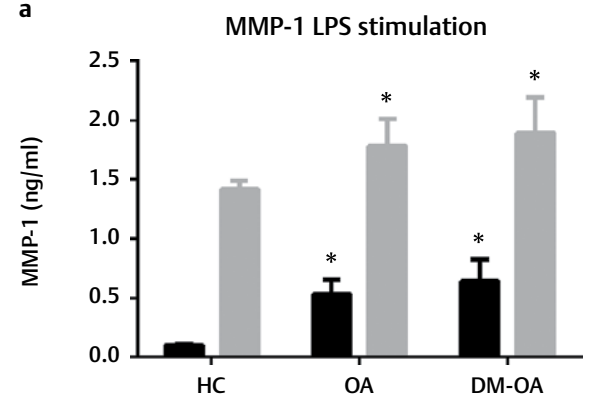

c

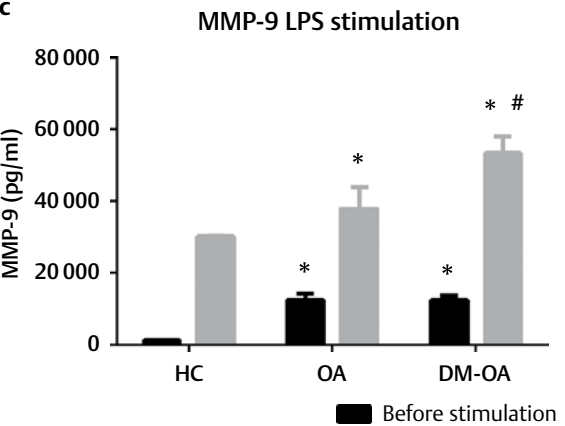

b

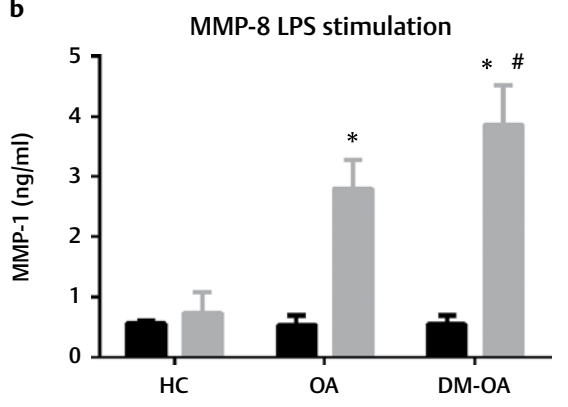

d

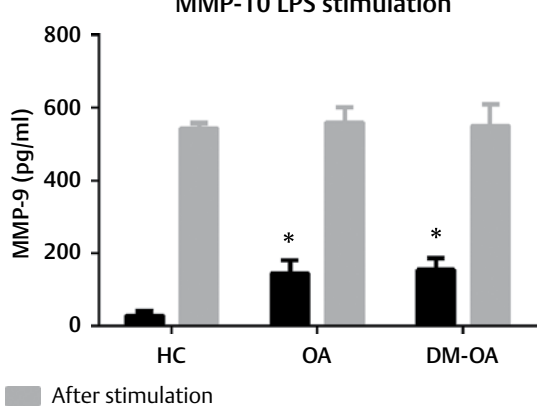

- Fig. 3 Role of PBMC in modulating MMPs expression profiles induced by LPS in HC, OA and DM-OA groups. Levels of MMP-1, $-8,-9,-10$ before and after $4 \mathrm{~h}$ stimulation with LPS were measured by ELISA. a MMP-1; $\mathbf{b}$ MMP-8; c MMP-9; $\mathbf{d}$ MMP-10. Each bar represents the mean \pm SD. ${ }^{*} \mathrm{P}<0.05$ vs. healthy control. \#P< 0.05 vs. OA group. p values result from a Tukey's multiple comparison test.

nificant differences in MMP-8, -9 and -10 protein levels among three groups. Hyperglycemia, a participant that led to excessive secretion of MMP-1 in serum and synovial fluid, is considered one of the reasons for facilitating the development and/or progression of OA.

Epidemiologic studies have established an association between inflammatory biomarkers and the occurrence of T2DM and complications. Low-grade inflammation is a common feature in subjects with T2DM [35, 36]. Synovitis has been considered as a secondary phenomenon not associated with systemic inflammation, but it is responsible for symptoms of $\mathrm{OA}$. Although inflammation is unlikely to play a primary role in initiating synovitis, growing evidences show that inflammation accelerates articular degeneration [37].

PBMCs consist of lymphocytes (T cells, B cells, NK cells) and monocytes, which can be extracted from whole blood using ficoll. There are also mononuclear cells in synovial fluid, called synovial fluid mononuclear cells (SFMCs). Limit to the amount of synovial fluid, we did not extract mononuclear cells from synovial fluid. Stimulation experiments with LPS are widely used to study the secretory function of PBMC. In response to LPS, the releases of MMP-1, $-8,-9$ and -10 were significantly increased at $4 \mathrm{~h}$ after stimulation of PMBC in three groups. In agreement with other studies [38], our presented results revealed no differences in the release of MMP-8 and -9 by PBMC between OA and DM-OA groups under unstimulated conditions. After $4 \mathrm{~h}$ stimulation with LPS however, PBMC released much more MMP- 8 and -9 in DM-OA group than in OA group or healthy controls. The extracellular glucose concentration, either increased or decreased, has been shown to directly affect some chondrocyte functions $[39,40]$.
MMPs inside synovial fluid are produced by chondrocytes and synoviocytes especially when stimulated by cytokines [41]. Our results also demonstrate that mononuclear cells have the ability to secrete MMP-1, $-8,-9$ and -10 . The level of MMPs in synovial fluid may be influenced by function of synovial fluid mononuclear cells to a certain degree. Although there are few mononuclear cells inside synovial fluid, the hyperfunction of mononuclear cells caused by inflammation might be one of the mechanisms of diabetes prone to $\mathrm{OA}$.

There were no significant differences of MMP-10 in three groups after LPS stimulation. It indicated that the function of PBMC secretion of MMP-10 did not change. MMP-10 in synovial fluid in DM-OA patients may rely on other inflammatory cytokines and MMPs for upregulation in chondrocytes and synovial fibroblasts, and may not be a malfunction of PBMC.

Our study had some limitations. First, our sample size was relatively small, especially the healthy control group, limiting its statistical power to detect existing associations. Moreover, the age of the healthy control group was much younger than OA and DM-OA groups. Whether age, blood glucose control and diabetes mellitus complication affect the level of MMPs in plasma or synovial fluid is unclear, it needs to be investigated in further study. Further research should evaluate whether diabetes promotes articular cartilage degradation, facilitating the development and/or progression of OA via of these MMPs in vivo and in vitro experiment.

In summary, high levels of MMP- $1,-7,-8,-9,-10$, and -12 in the synovial fluid might be one of important reasons that diabetes patients are more frequently suffered from OA. Inflammation-induced 
malfunction of mononuclear cells would stimulate MMP-8 and -9 secretion to various extents. Abnormal intrinsic regulatory mechanisms may activate other MMPs and produce amplification effect. Thus it can significantly increase MMPs activity and content. When the increment exceeds the degree, MMPs will lose the balance and further compromising cartilage integrity [42], thus promoting the degradation of articular cartilage matrix and inducing OA.

\section{Acknowledgments}

This project was supported by a grant from the National Natural Science Foundation of China (No. 81472089 and 81601219), China Postdoctoral Science Foundation (2015M582480 and 2017T100660), Natural Science Foundation of Guangdong Province (298763893) and Guangzhou Provincial Science and Technology Project of China (2014Y2-00084).

\section{Conflict of Interest}

No conflict of interest has been declared by the authors.

\section{References}

[1] Martel-Pelletier J, Boileau C, Pelletier JP et al. Cartilage in normal and osteoarthritis conditions. Best Pract Res Clin Rheumatol 2008; 22: 351-384

[2] White DK, Neogi T, Nguyen US et al. Trajectories of functional decline in knee osteoarthritis: The Osteoarthritis Initiative. Rheumatology (Oxford) 2016; 55: 801-808

[3] Ailixiding M, Aibibula Z, Iwata M et al. Pivotal role of Sirt6 in the crosstalk among ageing, metabolic syndrome and osteoarthritis. Biochem Biophys Res Commun 2015; 466: 319-326

[4] Askari A, Ehrampoush E, Homayounfar R et al. Serum insulin in pathogenesis and treatment of osteoarthritis. Med Hypotheses 2017; 99: 45-46

[5] Bourboulia D, Stetler-Stevenson WG. Matrix metalloproteinases (MMPs) and tissue inhibitors of metalloproteinases (TIMPs): Positive and negative regulators in tumor cell adhesion. Semin Cancer Biol 2010; 20: 161-168

[6] Goldring MB, Goldring SR. Osteoarthritis. ] Cell Physiol 2007; 213: 626-634

[7] Jackson MT, Moradi B, Smith MM et al. Activation of matrix metalloproteinases 2, 9, and 13 by activated protein $C$ in human osteoarthritic cartilage chondrocytes. Arthritis Rheumatol 2014; 66: 1525-1536

[8] Tio L, Martel-Pelletier J, Pelletier JP et al. Characterization of opticin digestion by proteases involved in osteoarthritis development. Joint Bone Spine 2014; 81: 137-141

[9] Heard B], Martin L, Rattner JB et al. Matrix metalloproteinase protein expression profiles cannot distinguish between normal and early osteoarthritic synovial fluid. BMC Musculoskelet Disord 2012; 13: 126

[10] Xue M, McKelvey K, Shen K et al. Endogenous MMP-9 and not MMP-2 promotes rheumatoid synovial fibroblast survival, inflammation and cartilage degradation. Rheumatology (Oxford) 2014; 53: 2270-2279

[11] Buhrmann C, Mobasheri A, Matis U et al. Curcumin mediated suppression of nuclear factor-KB promotes chondrogenic differentiation of mesenchymal stem cells in a high-density co-culture microenvironment. Arthritis Res Ther 2010; 12: R127
[12] Henrotin Y, Clutterbuck AL, Allaway D et al. Biological actions of curcumin on articular chondrocytes. Osteoarthritis and cartilage 2010; 18: $141-149$

[13] Courties A, Sellam J. Osteoarthritis and type 2 diabetes mellitus: What are the links? Diabetes Res Clin Pract 2016; 122: 198-206

[14] Ribeiro M, Lopez de Figueroa P, Nogueira-Recalde U et al. Diabetesaccelerated experimental osteoarthritis is prevented by autophagy activation. Osteoarthritis and cartilage 2016; 24: 2116-2125

[15] Berenbaum F. Diabetes-induced osteoarthritis: from a new paradigm to a new phenotype. Ann Rheum Dis 2011; 70: 1354-1356

[16] Yoshimura N, Muraki S, Oka H et al. Accumulation of metabolic risk factors such as overweight, hypertension, dyslipidaemia, and impaired glucose tolerance raises the risk of occurrence and progression of knee osteoarthritis: a 3-year follow-up of the ROAD study. Osteoarthritis Cartilage 2012; 20: 1217-1226

[17] American Diabetes Association. Diagnosis and classification of diabetes mellitus. Diabetes care 2014; 37: (Suppl 1): S81-S90

[18] Archer CW, Francis-West P. The chondrocyte. Int J Biochem Cell Biol 2003; 35: 401-404

[19] Itka O, Kukacka J, Krizkova S et al Matrix metalloproteinases. Curr Med Chem 2010; 17: 3751-3768

[20] Galasso O, Familiari F, De Gori M et al. Recent findings on the role of gelatinases (matrix metalloproteinase-2 and -9) in osteoarthritis. Adv Orthop 2012; 2012: 834208

[21] Catterall JB, Stabler TV, Flannery CR et al. Changes in serum and synovial fluid biomarkers after acute injury (NCT00332254). Arthritis Res Ther 2010; 12: R229

[22] Tchetverikov I, Lohmander LS, Verzijl N et al. MMP protein and activity levels in synovial fluid from patients with joint injury, inflammatory arthritis, and osteoarthritis. Ann Rheum Dis 2005; 64: 694-698

[23] Haller JM, Swearingen CA, Partridge D et al. Intraarticular matrix metalloproteinases and aggrecan degradation are elevated after articular fracture. Clin Orthop Relat Res 2015; 473: 3280-3288

[24] Hart D], Doyle DV, Spector TD. Association between metabolic factors and knee osteoarthritis in women: The Chingford study. J Rheumatol 1995; 22: 1118-1123

[25] Courties A, Sellam J, Berenbaum F. Metabolic syndrome-associated osteoarthritis. Curr Opin Rheumatol 2017; 29: 214-222

[26] Chadha R. Revealed aspect of metabolic osteoarthritis. J Orthop 2016; 13: 347-351

[27] Andereya S, Streich N, Schmidt-Rohlfing B et al. Comparison of modern marker proteins in serum and synovial fluid in patients with advanced osteoarthrosis and rheumatoid arthritis. Rheumatol Int 2006; 26: 432-438

[28] Rosa SC, Rufino AT, Judas FM et al. Role of glucose as a modulator of anabolic and catabolic gene expression in normal and osteoarthritic human chondrocytes. J Cell Biochem 2011; 112: 2813-2824

[29] Kevorkian L, Young DA, Darrah C et al. Expression profiling of metalloproteinases and their inhibitors in cartilage. Arthritis Rheum 2004; 50: 131-141

[30] Takaishi H, Kimura T, Dalal S et al. Joint diseases and matrix metalloproteinases: a role for MMP-13. Curr Pharm Biotechnol 2008; 9: 47-54

[31] Bauvois B. New facets of matrix metalloproteinases MMP-2 and MMP-9 as cell surface transducers: outside-in signaling and relationship to tumor progression. Biochim Biophys Acta 2012; 1825: 29-36

[32] Fernandes JC, Martel-Pelletier J, Pelletier JP. The role of cytokines in osteoarthritis pathophysiology. Biorheology 2002; 39: 237-246

[33] Tao Y, Qiu X, Xu C et al. Expression and correlation of matrix metalloproteinase-7 and interleukin-15 in human osteoarthritis. Int J Clin Exp Pathol 2015; 8: 9112-9118 
[34] Zeng GQ, Chen AB, Li W et al. High MMP-1, MMP-2, and MMP-9 protein levels in osteoarthritis. Genet Mol Res 2015; 14: 14811-14822

[35] Marques-Vidal P, Schmid R, Bochud M et al. Adipocytokines, hepatic and inflammatory biomarkers and incidence of type 2 diabetes. The CoLaus Study. PLoS One 2012; 7: e51768

[36] Calle MC, Fernandez ML. Inflammation and type 2 diabetes. Diabetes Metab 2012; 38: 183-191

[37] Pelletier JP, Martel-Pelletier J, Abramson SB. Osteoarthritis, an inflammatory disease: potential implication for the selection of new therapeutic targets. Arthritis Rheum 2001; 44: 1237-1247

[38] Death AK, Fisher EJ, McGrath KC et al. High glucose alters matrix metalloproteinase expression in two key vascular cells: potential impact on atherosclerosis in diabetes. Atherosclerosis 2003; 168 : 263-269

[39] Richardson S, Neama G, Phillips T et al. Molecular characterization and partial cDNA cloning of facilitative glucose transporters expressed in human articular chondrocytes; Stimulation of 2-deoxyglucose uptake by IGF-I and elevated MMP- 2 secretion by glucose deprivation. Osteoarthritis and cartilage 2003; 11: 92-101
[40] McNulty AL, Stabler TV, Vail TP et al. Dehydroascorbate transport in human chondrocytes is regulated by hypoxia and is a physiologically relevant source of ascorbic acid in the joint. Arthritis Rheum 2005; 52 : 2676-2685

[41] Nakamura H, Shibakawa A, Tanaka M et al. Effects of glucosamine hydrochloride on the production of prostaglandin E2, nitric oxide and metalloproteases by chondrocytes and synoviocytes in osteoarthritis. Clin Exp Rheumatol 2004; 22: 293-299

[42] Martel-Pelletier J, Welsch DJ, Pelletier JP. Metalloproteases and inhibitors in arthritic diseases. Best Pract Res Clin Rheumatol 2001; 15 : 805-829 